\title{
Puberty Blues and Pyogenic Granuloma In Females- A Report Of Two Cases
}

\author{
Afaf Zia ${ }^{1 *}$, Huda Zubair ${ }^{1}$, Shagufta Qadri ${ }^{2}$, Mukhtar Andrabi ${ }^{1}$, Masood Khan ${ }^{1}$ and Afshan Bey ${ }^{1}$ \\ ${ }^{1 *} \operatorname{Dr} Z$ A Dental College, Aligarh Muslim University, Aligarh, India \\ ${ }^{2}$ Department of Pathology, JNMCH, Aligarh Muslim University, Aligarh, India
}

Received: August 29, 2016; Accepted: September 12, 2016; Published: September 30, 2016

*Corresponding author: Afaf Zia, Assistant Professor, Department of Periodontology, Dr Z A Dental College, Aligarh Muslim University, Aligarh, India, 00919897789011,afafzia@gmail.com

\begin{abstract}
Pyogenic Granuloma is a commonly occurring tumor-like hyper plastic growth on the skin and the mucous membrane. There is markedly prominent angiogenesis which is a contributing factor to its typically red color. Its absolute etiological factors are still unknown, although minor trauma and endocrinal imbalances have known to play an important role. In the literature there is a well established relationship between the estrogen group of hormones and the angiogenetic factors like vascular endothelial growth factor and basic fibroblast growth factor. In this report, we have discussed and highlighted the apparent attribution of pyogenic granuloma to raised female sex hormones in pubertal young females of ages ranging from 12-18 years. In conclusion we suggest hormonal imbalance and pubertal changes can also be counted as an etiological factor for pyogenic granulomas in young females.
\end{abstract}

\section{Introduction}

Pyogenic Granuloma is considered to be the most common tumour of the oral cavity. It may be sessile or pedunculated, of various shades from pink to red or dark brown depending on its type or histogenesis. A ratio of 1:1.5 has been mentioned as the epidemiological occurrence among males and females ${ }^{1}$. Females of second decade being affected the most [2,3,4]. Although local irritating factors, hormonal disturbances (as in pregnancy) and poor oral hygiene are considered to be its etiological factors but its absolute causes are still obscure. In this report we discuss the occurrence of pyogenic granuloma in non-pregnant healthy young females of 12-18 years with good oral hygiene falling in the category of pubertal ages. Through this report we will shed some light on mildly raised estrogen group of hormones or other pubertal changes leading to such tumor like growth through various angiogenetic factors.

\section{Case Report 1}

A 15 year old female reported in the OPD of Dr Ziauddin Ahmad Dental College with a growth on her gums. The patient complained of painless slowly growing nodule on her gums since two weeks. History revealed that the swelling was pin point sized 2 weeks back which gradually grew large enough to bring her to seek medical care. The oral hygiene maintenance of the patient was commendable. There was no history of any kind of trauma in the recent past. The patient brushed twice daily with horizontal brushing strokes.

On examining clinically, there was a solitary growth seen with respect to the upper left lateral incisor. The growth involved the marginal gingival, was pedunculated, pink in color measuring about $1.8 \mathrm{~cm}$ in diameter, round in shape, with smooth surface. (Figure 1)

On the basis of history, clinical presentation and examination, a provisional diagnosis of pyogenic granuloma was made. An excisional biopsy was performed after the necessary investigations that are the complete blood picture, bleeding time and clotting time under local anaesthesia. The excisied tissue was sent for biopsy. (Figure 2) The patient wound healed uneventfully and was recalled every three months for one year. (Figure 3) Recall appointments till 1 year showed no recurrence of the lesion

The biopsy showed neocapillaries separated by edematous stroma infiltrated with inflammatory cells. Surface stratified epithelium was thickened and ulcerated focally confirming pyogenic granuloma. (Figure 4)

\section{Case Report 2}

A young female of 18 years reported to the periodontics OPD of Dr Ziauddin Ahmad Dental College with a swelling on her gums

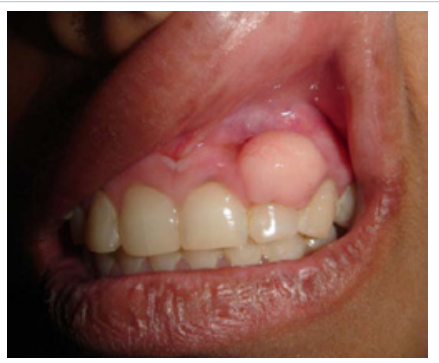

Figure 1: Preoperative picture showing growth with respect to upper left lateral incisor 
that had gradually increased within a span of three weeks. She complained of mild pain and difficulty in speaking or chewing. She gave no history of trauma. The patients brushed in vertical strokes twice a day.

Intraoral examination revealed a large firm polyploid mass with a broad base and smooth pink surface, measuring $10 \times 7$ $\mathrm{mm}$ in size in relation to the gingiva of mandibular right canine. The peculiar feature of this growth was its unusual growth on the lingual surface of the lower anteriors. Over all calculus and plaque accumulation was negligible. Other systemic involvement was non contributory with no lymphadenopathy. (Figure 5a and 5b)

After preliminary investigation which included complete hemogram, bleeding time and clotting time, the growth was excised under local anaesthesia and sent for biopsy. (Figure 6) The patient was kept on recall visits every month for a year. (Fig 7) There was no recurrence of the lesion and the patient showed excellent recovery.

The histopathological report showed lobular growth lined by stratified squamous epithelium, while underlying deeper tissues shows proliferating capillaries and dense inflammatory infiltrates like neutrophils, lymphocyte and plasma cells.

\section{Discussion}

Pyogenic granuloma represents an exuberant connective tissue proliferation to a known stimulus or injury [5]. Originally it was believed to be a pyogenic growth but there is enough evidence against it as it does not contain pus neither is it a granuloma in a strict sense. Hamid Jaferzadeh, et al. have mentioned in their review that about one third of the cases occur after any trauma

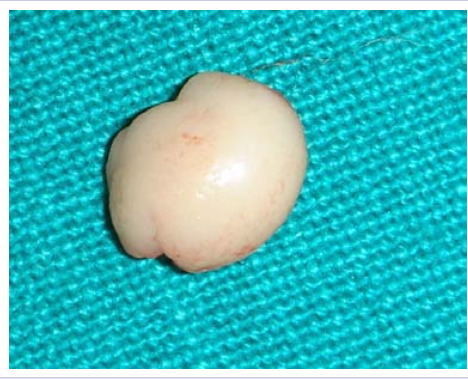

Figure 2: Excised tissue.

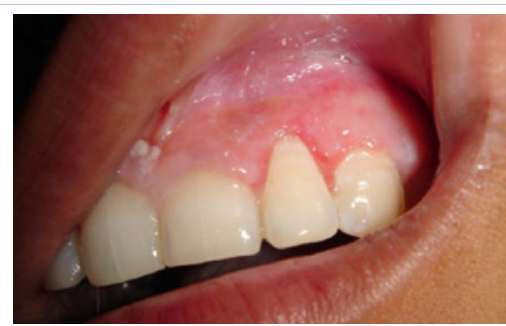

Figure 3: Postoperative picture showing complete healing after excision.

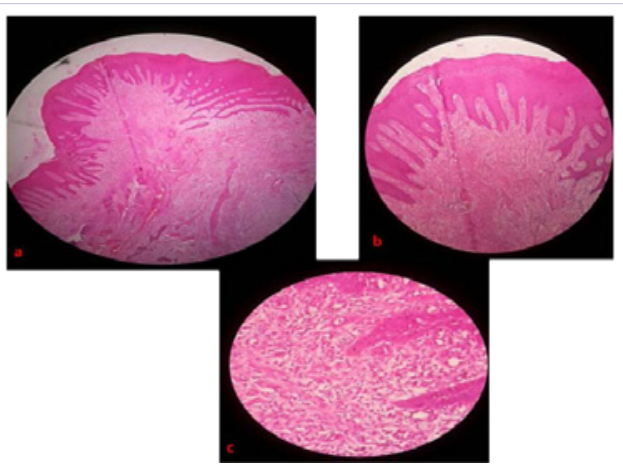

Figure 4: Histological section of the growth under 10X (4a), 40X (4b) $\& 100 \mathrm{X}(4 \mathrm{c})$
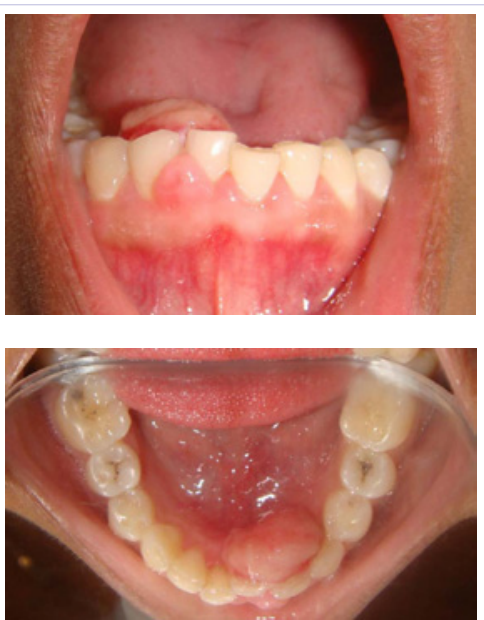

Figure 5: Preoperative picture showing the lesion labially (5a) and lingually $(5 b)$
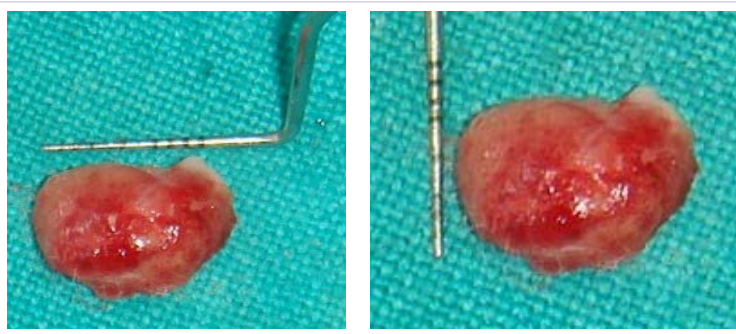

Figure 6: Excised tissue

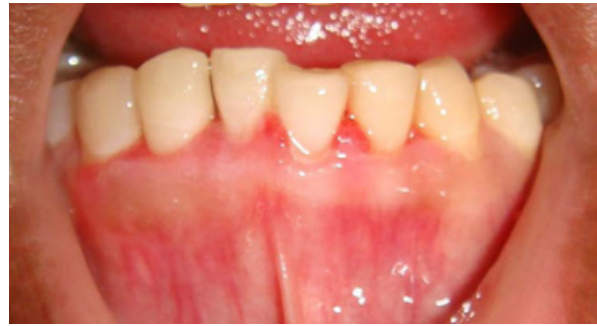

Figure 7: Postoperative picture showing complete healing after excision 
or injury [6]. According to Vilmann, et al. majority of the pyogenic granulomas are found on the marginal gingiva with only $15 \%$ of the tumors on the alveolar part [8]. Regezi, et al. mention that it is seen mostly common in the second life of females $[5,9]$. It occurs more commonly in the females and seen in upto $5 \%$ of pregnancies. Yuank, et al. has demonstrated a strong relationship between the angiogenetic factors especially vascular endothelial growth factor and basic fibroblastic growth factors and pyogenic granuloma [7]. There is also an undisputed relationship between these factors and their directly dependant relationship between female sex hormones. The gradual but marked rise in the levels of female sex hormones; associated with puberty and specifically menarche; especially the estrogen group of hormones may play an important role in initiating or promoting the occurrence of pyogenic granuloma. In a study done by Michael D. Graubert, et al. they demonstrated that VEGF expression peaks during days 1 to 3 of the endometrial cycle. In addition, they provide evidence that physiological hypoxia in conjunction with TGF- $\alpha$ and IL-1 $\beta$ are most likely responsible for the induction and sustained levels of VEGF during menstruation [11].

In the cases presented here the female patients were of the age group corresponding to the pubertal age contrary to the common incidence in the second decade of life in females. Also the presenting females were not pregnant and rather healthy. The oral hygiene in all the above cases does not direct the etiology towards local irritation or poor oral hygiene. There was also absence of history of trauma. Thus the abrupt hormonal rise in corresponding to the pubertal spurt might have played a role in the development of pyogenic granuloma in these cases.

Bhaskar SN, Jacoway JR observed in their study [10] that pyogenic granuloma is partly or completely covered by parakeratinased or non-keratinised stratified squamous epithelium. Major bulk of the lesion is formed by a lobulated or a non lobulated mass of angiomatous tissue. Usually, lobulated lesions are composed of solid endothelial proliferation or proliferation of capillary sized blood vessels. The amount of collagen in the connective tissue of pyogenic granuloma is usually sparse. Surface can be ulcerated and in such ulcerated lesions, edema was a prominent feature and the lesion is infiltrated by plasma cells, lymphocytes and neutrophils.

Various other benign soft tissue lesions need to be differentiated from pyogenic granuloma. Few to name include peripheral giant cell granuloma, pregnancy tumor; conventional granulation tissue etc [6].
Surgical excision is the treatment of choice. After surgical excision of gingival lesions, curettage of underlying tissue is recommended. Bhaskar SN, Jacoway JR has reported one recurrence after conservative excision with a recurrence rate of $15.8 \%$. They also stated that pyogenic granuloma lacks infiltrative or malignant potential [10].

\section{Conclusion}

Pyogenic granuloma is a common oral lesion occurring in females. Although numerous factors are responsible for its causes, its proper diagnosis and management is essential to prevent its recurrence. In this case report we highlighted the role of hormonal imbalance in the absence of local deposits as one of the cause of pyogenic granuloma in healthy young females of pubertal age and their successful management.

\section{References}

1. Epivatianos A, Antoniades D, Zaraboukas T, Zairi E, Poulopoulos A, Kiziridou A, et al. Pyogenic granuloma of the oral cavity: comparative study of its clinicopathological and immunochemical features. Pathol Int J. 2005;55:391-397.

2. Eversole LR. Clinical outline of oral pathology: diagnosis and treatment. 3rd ed, BC Decker, Hamilton. 2002; 113-114.

3. Neville BW, Damm DD Allen CM, Bouqout JE. Oral and maxillofacial pathology. 2nd ed, WB Saunders, Philadelphia, 2002; 437-495

4. Regezi JA, Sciubba JJ, Jordan RCK. Oral pathology: clinical pathologic considerations. 4th ed, WB Saunders, Philadelphia, 2003;115-116

5. Regezi JA, Sciubba JJ, Jordan RCK. Oral pathology: clinical pathologic considerations .6th edn, WB Saunders, Philadelphia, 2003; 259-260.

6. Hamid J, Majid S, Nooshin M. Oral Pyogenic Granuloma Review. J Oral Sci. 2006;48(4):167-175.

7. Yuan K, Jin YT, Lin MT. Expression of Tie-2, angiopoietin-1, angiopoietin-2, ephrinB2 and EphB4 in pyogenic granuloma of human gingiva implicates their roles in inflammatory angiogenesis. J Periodontal Res. 2000;35:165-171.

8. Vilmann A, Vilmann P, Vilmann H. Pyogenic granuloma: evaluation of oral conditions. British J Oral Maxillofac Surg. 1986;24(5):376-382.

9. Lawoyin J, Arotiba J, Dosumu O. Oral pyogenic granuloma: a review of 38 cases from Ibadan, Nigeria. Br J Oral Maxillofac Surg. 1997;35(3):185-189.

10. Bhaskar SN, Jacoway JR. Pyogenic granuloma - clinical features, incidence, histology, and result of treatment: Report of 42 cases. J Oral surg. 1966;24:391-398.

11. Michael D.Graubert, Maria Asuncion Ortega, Bruce Kessel, Joseph F. Mortola, M. Luisa Iruela-Arispe. Vascular Repair after Menstruation Involves Regulation of Vascular Endothelial Growth Factor-Receptor Phosphorylation by sFLT-1. Am J Path. 2001;158(4):1399-1410. 